\title{
ARTICLE \\ Prefrontal cortical activation during working memory task anticipation contributes to discrimination between bipolar and unipolar depression
}

\author{
Anna Manelis ${ }^{1}$, Satish lyengar ${ }^{2}$, Holly A. Swartz ${ }^{1}$ and Mary L. Phillips ${ }^{1}$
}

\begin{abstract}
Distinguishing bipolar disorder (BD) from major depressive disorder (MDD) is clinically challenging, especially during depressive episodes. While both groups are characterized by aberrant working memory and anticipatory processing, the role of these processes in discriminating BD from MDD remains unexplored. In this study, we examine how brain activation corresponding to anticipation of and performance on easy vs. difficult working memory tasks with emotional stimuli contributes to discrimination among BD, MDD, and healthy controls (HC). Depressed individuals with BD $(n=18), \operatorname{MDD}(n=23)$, and HC $(n=23)$ were scanned while performing a working memory task in which they had to first anticipate performance on 1-back (easy) or 2-back (difficult) tasks with happy, fearful, or neutral faces, and then, perform the task. Anticipation-related and task-related brain activation was measured in the whole brain using functional magnetic resonance imagining. We used an elastic-net regression for variable selection, and a random forest classifier for BD vs. MDD classification. The former selected the activation differences (1-back minus 2-back) in the lateral and medial prefrontal cortices (PFC) during task anticipation and performance on the working memory tasks with fearful and neutral faces as variables relevant for BD vs. MDD classification. BD vs. MDD were classified with $70.7 \%$ accuracy $(p$ $<0.01$ ) based on the neuroimaging measures alone, with $80.5 \%$ accuracy $(p=0.001)$ based on clinical measures alone, and with $85.4 \%$ accuracy $(p<0.001)$ based on clinical and neuroimaging measures together. These findings suggest that PFC activation during working memory task anticipation and performance may be an important biological marker distinguishing BD from MDD.
\end{abstract}

Neuropsychopharmacology (2020) 45:956-963; https://doi.org/10.1038/s41386-020-0638-7

\section{INTRODUCTION}

Distinguishing bipolar disorder (BD) from major depressive disorder (MDD) remains challenging in clinical practice because diagnostic criteria for depressive episodes in the context of BD and MDD are identical [1]. Neuroimaging studies directly comparing depressed individuals with $\mathrm{BD}$ and MDD may help to identify objective neurobiological markers for these disorders, thus improving symptomatic and functional outcomes for affected individuals [2].

Working memory is a limited-capacity system used to maintain, manipulate, and update information online [3]. The difficulty of a working memory task is determined by the amount of information that an individual needs to process at one time. A more difficult vs. easy task requires processing of more information, recruits additional neurocognitive resources, and results in behavioral performance worsening and greater activation in a so-called working memory network consisting of prefrontal cortical regions (PFC), anterior cingulate cortex (ACC), posterior parietal cortex, and some medial temporal and striatal regions [3-8]. Damage to the neural substrates of the working memory network leads to impairment in learning, reasoning, and decision-making [4, 9].

Task performance is preceded by task anticipation. During task anticipation, individuals prepare for a task by recollecting task-related rules and forming expectations related to the task's difficulty and emotional valence. Anticipation-related brain activation may interact with performance-related brain activation to ensure optimal brain dynamics and resource usage. For example, PFC and ACC are involved during anticipation of emotional stimuli [10]. Anticipatory activation in these regions predicted reaction time (RT) during problem-solving tasks [11]. Activation in the left ventrolateral PFC (VLPFC) and dorsal anterior cingulate cortex (dACC) increased when a more difficult vs. easy task was performed, but decreased when a more difficult task was anticipated [7]. This activation pattern might be related to "clearing of" online storage of information to free neural and cognitive resources prior to performing a difficult working memory task [7].

Individuals with mood disorders often suffer from cognitive dysfunction, especially during depressive episode [12-19] that maybe related to aberrant functioning of the regions described above [19-24]. Depressed individuals also show strong negative bias when anticipating future events and experiences [25, 26]. Given that previous studies have demonstrated that individuals with $\mathrm{BD}, \mathrm{MDD}$, and $\mathrm{HC}$ have different activation patterns in ACC and PFC during working memory task performance $[24,27]$ and also during anticipation of monetary reward and loss [28, 29], it is possible that the three groups may also differ during anticipation of easy vs. difficult working memory tasks. Even though in real life people are constantly involved in evaluating the difficulty of

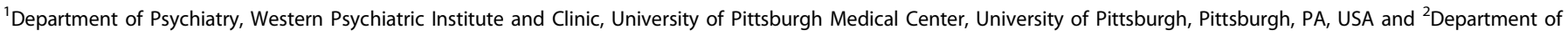
Statistics, University of Pittsburgh, Pittsburgh, PA, USA

Correspondence: Anna Manelis (anna.manelis@gmail.com)

Received: 17 September 2019 Revised: 3 February 2020 Accepted: 10 February 2020

Published online: 18 February 2020 
upcoming tasks, neural correlates associated with this process are largely underexplored. Meanwhile, task difficulty anticipation may play a critical role in cognitive and psychosocial functioning because misappraisal of task difficulty could result in poorly informed behaviors that might, in turn, impair functioning.

In this study, we aimed to examine the differences in anticipatory processing characterizing $\mathrm{BD}, \mathrm{MDD}$, and $\mathrm{HC}$, and to shed light on whether cognitive dysfunction in depression is related to the decreased ability to perform the task, decreased ability to prepare for the task, or both. Given that processing of emotion-independent stimuli may be differentially biased and altered by emotion-dependent cognition in depressed individuals [30], we used affective stimuli such as happy, fearful, and neutral faces as stimuli in a Cued Emotional N-back Task that examined anticipation of and performance on an easy (1-back) vs. difficult (2-back) working memory tasks in depressed individuals with BD, those with MDD and HC.

The differences (or deltas $([\boldsymbol{\Delta}])$ between easy vs. difficult tasks in RT $(\Delta R T)$, accuracy ( $\triangle$ accuracy), brain activation during task anticipation ( $\Delta$ cue), and task performance ( $\Delta$ task)) were calculated as they may be indicative of the amount of the additional neurocognitive resources required to perform a difficult vs. easy task. The interaction between $\Delta$ cue and $\Delta$ task, and $\Delta R T$ and $\Delta$ accuracy, may represent a trade-off between resource usage, freeing, and allocation prior to and during the task. These values together with behavioral and clinical variables were used in a multinomial elastic-net-regularized regression to identify variables contributing to BD vs. MDD vs. HC classification [31]. The predictive ability of selected variables was then validated using the random forest classifier [32].

\section{METHODS}

\section{Participants}

The study was approved by the University of Pittsburgh and Carnegie Mellon University (CMU) Institutional Review Boards. Participants were recruited from community, universities, counselling, and medical centers. Written informed consent was obtained from all participants. Participants were right-handed, fluent in English, and matched on age, gender, and IQ. HC had no personal or family history of psychiatric disorders. Symptomatic individuals met DSM-5 criteria either for major depressive (MDD) or bipolar (BD) disorders, and were depressed at scan (Hamilton Rating Scale for Depression (HRSD-25) [33] score >14). Of 21 individuals with BD, one did not finish the scan, one had low accuracy, and one had excessive motion (>4 mm). Of $25 \mathrm{HC}$, one had excessive motion, and one did not follow task instructions. Of 24 individuals with MDD, one participant had low accuracy. These six individuals were removed from the analyses. The final data set consisted of 64 participants: 18 with BD (BD Type I=3, Type II=15), 23 with MDD, and $23 \mathrm{HC}$.

\section{Clinical assessment}

All diagnoses were made by a trained clinician and confirmed by a psychiatrist according to DSM-5 criteria using MINI7.0 [34]. Exclusion criteria included a history of head injury, metal in the body, pregnancy, claustrophobia, neurodevelopmental disorders, systemic medical illness, premorbid $\mathrm{IQ}<85$ as per the National Adult Reading Test (NART) [35], current alcohol/drug abuse, the Young Mania Rating Scale (YMRS) [36] score >10 at scan, and meeting criteria for any psychotic-spectrum disorder. We also collected the following measures: current depression symptoms using the HDRS-25 [33], current mania symptoms using the YMRS [36], lifetime depression, hypo/mania spectrum symptomatology, mixed irritability and mixed impulsivity using the mood spectrum self-report questionnaire (MOODS-SR) [37], state/trait anxiety using the State-Trait Anxiety Inventory (STAIY1/STAIY2) [38], and anhedonia using the Snaith-Hamilton Pleasure Scale (SHAPS) [39]. A total psychotropic medication load was calculated for each participant, with greater numbers and doses of medications corresponding to a greater medication load [29, 40]. Table 1 reports means, standard errors, and group statistics for participants' demographic and clinical characteristics.

\section{Behavioral paradigm}

The Cued Emotional N-back Task modeled after Manelis and Reder $[7,41]$ included a total of 48 blocks of 15 trials, each with 8 blocks per task condition: 1- and 2-back with fearful, neutral, and happy faces. Face stimuli were taken from the NimStim [42] and the Karolinska Directed Emotional Faces [43] databases, and were presented on the white background in the oval frame limiting the background (Fig. 1). The trials in a block were separated with 500-, 750-, or 1000-ms intertrial intervals randomly inserted between the trials. The blocks were separated with $6-7.5 \mathrm{~s}$ of rest. The $\mathrm{n}$ back task required participants to determine whether the face currently presented on the screen matched the face presented $n$ faces earlier in the sequence. Matching faces were called targets, and all other faces-nontargets. Participants responded by pressing a button with the index finger on one hand for targets and on the other hand for nontargets. A hand assignment was counterbalanced across participants.

Each block of trials was preceded with the 6-s cue screen containing information about the upcoming $n$-back condition (1back/2-back) and a symbol indicating the emotional valence of upcoming stimuli [44] whose emotions were never intermixed within a block (Fig. 1). A straight line predicted a neutral emotional expression, a concave curve resembling a smile predicted a happy emotional expression, and a convex curve predicted fearful faces. During cue presentation, participants were instructed to anticipate the $n$-back condition and face emotion specified by the cue that always matched the task difficulty and emotional valence of the block of trials. Subjects underwent extensive practice outside the scanner to ensure high accuracy on the task inside the scanner.

Neuroimaging data acquisition

All neuroimaging data were collected at the Scientific Imaging and Brain Research Center at CMU using a Siemens Verio $3 \mathrm{~T}$ scanner with a 32-channel coil. The fMRl data were collected using a multiband sequence (factor $=4, T R=1500 \mathrm{~ms}$, resolution $=2 \times 2 \times$ $2 \mathrm{~mm}, 68$ slices, $\mathrm{FOV}=220, \mathrm{TE}=30 \mathrm{~ms}$, and flip angle $=72^{\circ}$ ). Highresolution T1w images were collected using the MPRAGE sequence $(\mathrm{TR}=2300 \mathrm{~ms}$, resolution $=1 \times 1 \times 1 \mathrm{~mm}, 176$ slices, $\mathrm{FOV}=256$, $\mathrm{TE}=1.97 \mathrm{~ms}$, and flip angle $=9^{\circ}$ ). Two spin-echo images were acquired with opposite phases (i.e., $\mathrm{AP}$ and $\mathrm{PA}$ ) encoding directions $(\mathrm{TR}=2240 \mathrm{~ms}$, resolution $=2 \times 2 \times 2 \mathrm{~mm}, 68$ slices, $\mathrm{FOV}=220, \mathrm{TE}=$ $76.4 \mathrm{~ms}$, and flip angle $=90^{\circ}$ ) for distortion correction.

\section{Data analyses}

Clinical data analysis. The demographic and clinical characteristics were compared among groups using a one-way ANOVA or chi-square test, as appropriate. BD and MDD were compared using a $t$ test. One missing trait anxiety score was imputed using the R package "mice" (https://cran.r-project.org/web/packages/mice/ mice.pdf).

Behavioral data analysis. A three-way ANOVA, with group (BD/MDD/HC) as a between-subject factor, and $n$-back (1-/2-back) and emotional conditions (happy/fearful/neutral faces) as withinsubject factors, was conducted using the "sjstats" package in R (https://cran.r-project.org/web/packages/sjstats/index.html).

\section{Neuroimaging data analysis}

Preprocessing: The quality of the neuroimaging data was examined using the MRIQC [45]. Data were preprocessed using fMRIPprep [46] (see Supplemental Materials). In short, the anatomical images (T1w) were corrected for intensity nonuniformity. Brain surfaces were reconstructed using recon-all (FreeSurfer6.0.1) [47]. 
Table 1. Demographic and clinical characteristics.

\begin{tabular}{|c|c|c|c|c|c|}
\hline & BD & MDD & $\mathrm{HC}$ & ANOVA/chi-square & $t$ test $\mathrm{BD}$ vs. MDD \\
\hline$N$ & 18 & 23 & 23 & & \\
\hline Gender (number of females) & 13 & 17 & 16 & ns & \\
\hline Age (years) & $25.88(1.3)$ & $25.8(1.5)$ & $24.7(1.2)$ & $\mathrm{F}(2,61)=0.24, p=0.79$ & $\mathrm{t}(39)=0.04, p=0.97$ \\
\hline IQ (NART) & $109.68(1.6)$ & $113.1(1.1)$ & $110.07(1.2)$ & $\mathrm{F}(2,61)=2.17, p=0.12$ & $\mathrm{t}(39)=-1.8, p=0.08$ \\
\hline Current depressive episode duration (in weeks) & $14.2(4.8)$ & $16.2(4.3)$ & na & na & $\mathrm{t}(39)=-0.3, p=0.76$ \\
\hline Number of lifetime mood episodes & $7.1(0.7)$ & $6.04(0.9)$ & na & na & $\mathrm{t}(39)=0.9, p=0.4$ \\
\hline Number of lifetime episodes of depression & $4.3(0.5)$ & $6.04(0.9)$ & na & na & $\mathrm{t}(39)=-1.5, p=0.15$ \\
\hline $\begin{array}{l}\text { Number of lifetime episodes of } \\
\text { - Mania } \\
\text { - Hypomania }\end{array}$ & $\begin{array}{l}0.33(0.2) \\
2.4(0.4)\end{array}$ & 0 & na & na & na \\
\hline Illness onset (year of age) & $17.3(0.8)$ & $19.2(1.6)$ & na & na & $\mathrm{t}(39)=-0.95, p=0.35$ \\
\hline Current depression severity (HRSD-25) & $27.11(1.5)$ & $21.5(1.1)$ & $1.3(0.3)$ & $\mathrm{F}(2,61)=177.39, p<0.001$ & $\mathrm{t}(39)=3.05, p=0.004$ \\
\hline Current mania severity (YMRS) & $3.33(0.6)$ & $1.7(0.3)$ & $0.43(0.2)$ & $\mathrm{F}(2,61)=13.73, p<0.001$ & $\mathrm{t}(39)=2.46, p=0.02$ \\
\hline State anxiety (STAIY1) & $52.56(2.4)$ & $51.5(1.8)$ & $26.26(1.1)$ & $\mathrm{F}(2,61)=74.7, p<0.001$ & $\mathrm{t}(39)=0.35, p=0.7$ \\
\hline Trait anxiety (STAIY2) & $61.56(3.1)$ & $60.9(1.8)$ & $30.78(1.2)$ & $\mathrm{F}(2,61)=79.38, p<0.001$ & $\mathrm{t}(39)=0.2, p=0.85$ \\
\hline Anhedonia (SHAPS) & $34.5(2)$ & $32.26(0.9)$ & $18(0.8)$ & $\mathrm{F}(2,61)=52.39, p<0.001$ & $\mathrm{t}(39)=1.08, p=0.3$ \\
\hline Lifetime depression (MOODS-SR) & $21.78(0.6)$ & $20.09(0.6)$ & $1.04(0.3)$ & $\mathrm{F}(2,61)=514.14, p<0.001$ & $\mathrm{t}(39)=1.93, p=0.06$ \\
\hline Lifetime mania (MOODS-SR) & $18.61(1)$ & $9.17(1.2)$ & $4.48(0.8)$ & $\mathrm{F}(2,61)=44.56, p<0.001$ & $\mathrm{t}(39)=5.8, p<0.001$ \\
\hline Lifetime mood mixed instability (MOODS-SR) & $4.11(0.5)$ & $1.7(0.4)$ & $0.17(0.1)$ & $\mathrm{F}(2,61)=31.54, p<0.001$ & $\mathrm{t}(39)=3.96, p<0.001$ \\
\hline Lifetime irritability (MOODS-SR) & $4.5(0.3)$ & $2.74(0.3)$ & $0.39(0.1)$ & $F(2,61)=63.17, p<0.001$ & $\mathrm{t}(39)=4.04, p<0.001$ \\
\hline Number of participants taking antidepressants & 10 & 12 & 0 & & \\
\hline Number of participants taking mood stabilizers & 4 & 0 & 0 & & \\
\hline Number of participants taking antipsychotics & 0 & 0 & 0 & & \\
\hline Number of participants taking benzodiazepines & 5 & 3 & 0 & & \\
\hline Number of participants taking stimulants & 1 & 0 & 0 & & \\
\hline A mean number of psychotropic medications & $1.22(0.2)$ & $0.78(0.2)$ & $0(0)$ & $\mathrm{F}(2,61)=18.03, p<0.001$ & $\mathrm{t}(39)=1.6, p=0.1$ \\
\hline A mean total medication load & $1.5(0.2)$ & $1.17(0.3)$ & $0(0)$ & $\mathrm{F}(2,61)=15.1, p<0.001$ & $\mathrm{t}(39)=0.88, p=0.4$ \\
\hline Number of participants taking one psychotropic medication & 6 & 6 & 0 & & \\
\hline Number of participants taking two psychotropic medication & 8 & 6 & 0 & & \\
\hline
\end{tabular}

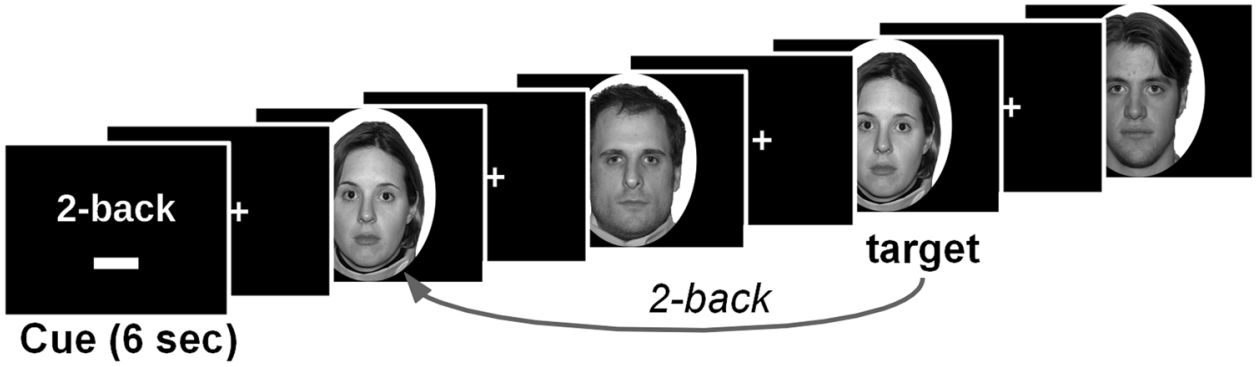

Fig. 1 N-back task. An example of a 2-back block with neutral emotional faces.

Brain tissue segmentation of cerebrospinal fluid (CSF), white matter (WM), and gray matter was performed on the brain-extracted T1w using FAST [48]. Susceptibility distortions of BOLD images were estimated based on two spin-echo images with opposing phaseencoding directions using 3dQwarp of AFNI [49]. The BOLD images were co-registered to the T1w images using bbregister [50] and motion corrected using MCFLIRT [51]. Automatic removal of motion artifacts was achieved using independent component analysis (ICA-AROMA) [52]. Further preprocessing included removing the effects of CSF, WM, and non-steady-state volumes and high-pass temporal filtering $(90 \mathrm{~s})$.

Subject-level analysis: The preprocessed and registered-to-MNIspace BOLD images served as an input to a subject-level analysis.
A hemodynamic response was modeled using a gamma function. A subject-level general linear model included 13 explanatory variables: 2 levels of task difficulty (1-back/2-back), 3 emotional conditions (happy/neutral/fearful), 2 task conditions (task anticipation/task performance), and motor responses. The former 12 variables were modeled as blocks. The motor response was modeled as the last $200 \mathrm{~ms}$ of each valid trial. The 1-minus-2-back contrasts were computed for task anticipation and performance across all emotions.

Group-level analysis: Two group-level analyses (1-minus-2-back during task anticipation, and 1-minus-2-back during task performance) were conducted across all subjects and all emotional conditions in the whole brain using the randomise tool [53] for 
Table 2. Elastic-net regression coefficients for multinomial and binomial elastic regressions.

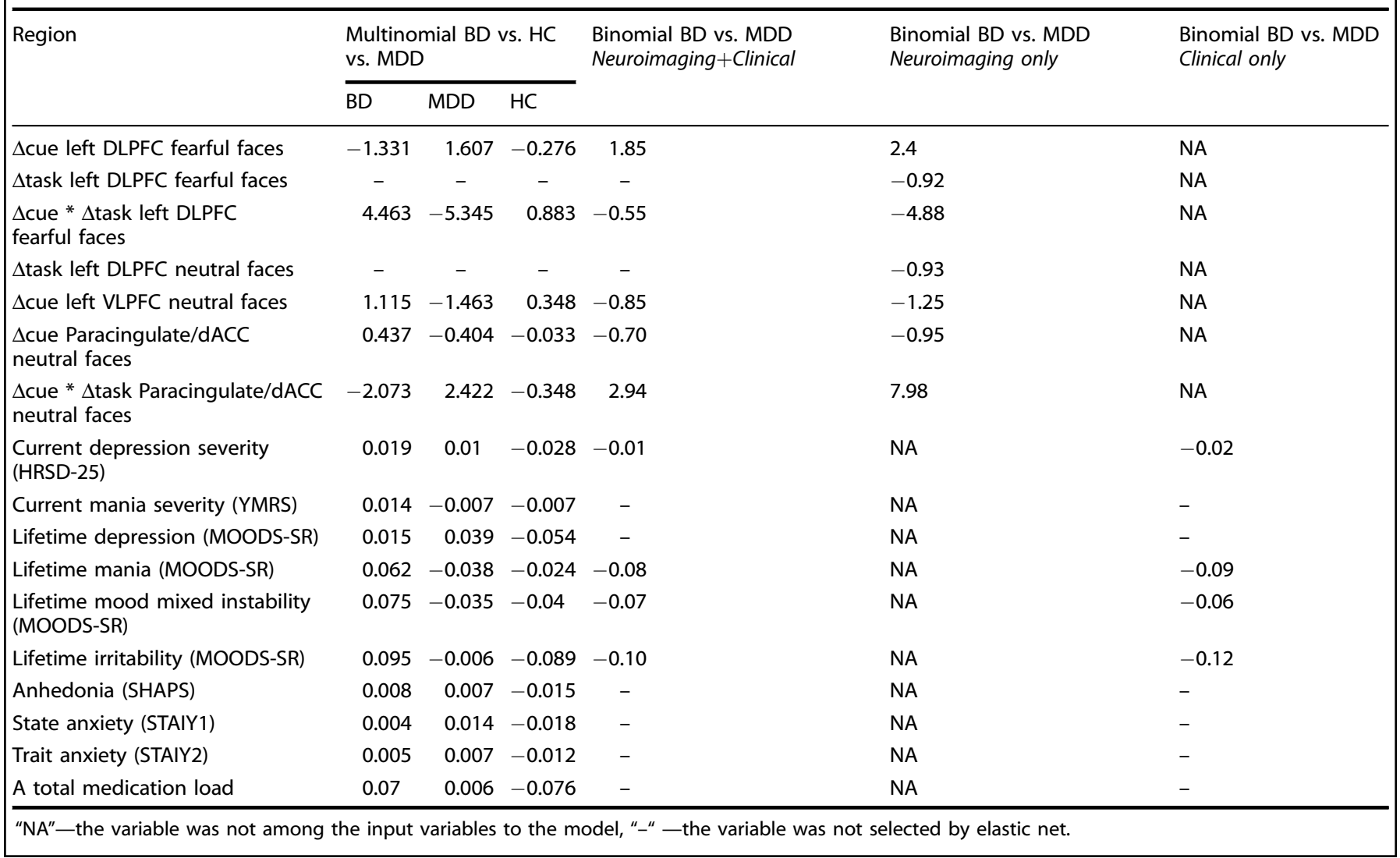

nonparametric permutation inference with 5000 permutations, Threshold-Free Cluster Enhancement correction and FWEcorrected $p$-values were $<0.05$. Subjects' age, gender, and IQ were used in the models as covariates. A conjunction analysis was used to map the brain regions sensitive to anticipated task difficulty (1-back $>2$-back or 2-back $>1$-back during anticipation) on the working memory network (2-back $>1$-back during task performance). The percent signal changes were then extracted from the regions identified in the conjunction analysis for each subject for each n-back, emotion, and anticipation/task condition, and then $\Delta$ cue and $\Delta$ task (1-minus-2-back differences) were calculated for all these conditions. Functional localization was determined using the Harvard-Oxford cortical and subcortical structural atlases.

Multinomial regression with elastic-net regularization: The measures of $\Delta$ cue, $\Delta$ task, $\Delta c$ cue $^{*} \Delta$ task interaction, $\Delta \mathrm{RT}, \Delta$ accuracy, $\Delta R T^{*} \Delta$ accuracy interaction for the same emotional condition, and current depression and mania severity, lifetime depression, mania, instability and irritability, state and trait anxiety, anhedonia, and a total medication load were used as inputs to the multinomial (BD vs. MDD vs. $\mathrm{HC}$ ) and binomial (BD vs. MDD) elastic-net-regularized regression models (the elastic-net penalty $a=0.5$ ) implemented using the R package "glmnet" [31]. Elastic net is a regularization and variable selection method that combines the L1 norm (for model sparsity) and L2 norm (for model robustness) into a penalized model [31] used when the model has too many predictors relative to the number of observations. A lambda.1se computed across 5000 repetitions of the elastic-net algorithm with tenfold cross-validation was used to predict subjects' diagnostic groups. The binomial elastic net to discriminate BD and MDD diagnoses was run on clinical and neuroimaging, clinical-only, and neuroimaging-only variables. Table 2 reports the model's coefficients. Despite a progress in developing test statistics for other types of elastic-net regression [54, 55], procedures for calculating $p$-values for multinomial/binomial elastic nets are not yet available. In order to validate the elastic-net results and estimate a classification accuracy, we used the variables selected by elastic net in a random forest classification analysis using the R package "randomForest" [32] (https://cran.r-project.org/web/ packages/randomForest/randomForest.pdf) with ntrees $=10000$. The significance was determined using the $\mathrm{R}$ package 'rfUtilities' (https://cran.r-project.org/web/packages/rfUtilities/index.html) with ntrees $=10000$ and 5000 permutations (see Supplemental Methods).

\section{RESULTS}

Clinical

$\mathrm{BD}, \mathrm{MDD}$, and $\mathrm{HC}$ groups did not differ from each other in age, $I Q$, or gender composition (Table 1). Individuals with BD and MDD were significantly more symptomatic than $\mathrm{HC}(p<0.05)$. Individuals with BD had more severe symptoms of depression, hypo/ mania, mood instability and irritability lifetime, had more subthreshold symptoms of mania, and were significantly more depressed at scan $(p<0.05)$ than those with MDD.

\section{Behavioral}

A group $\times n$-back $\times$ emotion ANOVA revealed the main effect of task difficulty on both accuracy $(\mathrm{F}(1,365)=201.7, p<0.001$, partial $\left.\eta^{2}=0.356\right)$ and RT $\left(F(1,365)=118.9, p<0.001\right.$, partial $\left.\eta^{2}=0.346\right)$. Participants were slower and less accurate for 2-back vs. 1-back task (Fig. 2), thus confirming that the 2-back was more difficult than the 1-back task. Neither accuracy nor RT were affected by group, emotional condition, or interactions between group, task difficulty, and emotional condition ( $p$-value $>0.05$ ). 

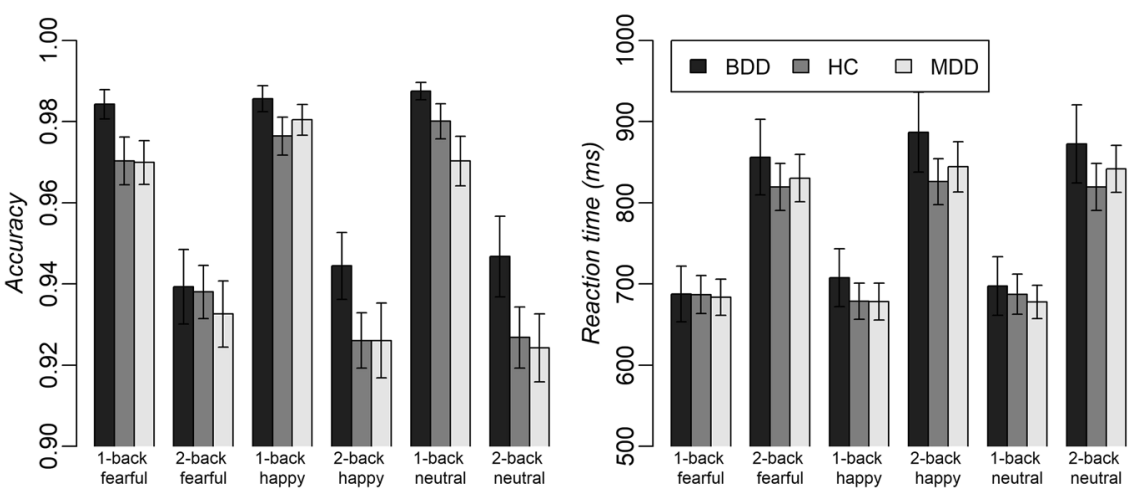

Fig. 2 Behavioral results. Accuracy and RT for 1- and 2-back tasks with fearful, happy, and neutral faces.

Neuroimaging: 1- vs. 2-back contrast across subjects and emotional conditions

Bilateral VLPFC, DLPFC, paracingulate/dACC, insula, cerebellum, left posterior parietal cortices, right thalamus, and right caudate regions showed greater activation during performance on 2-back vs. 1-back task across all emotional conditions, while central and parietal opercular, frontal polar, and posterior cingulate cortices showed greater activation during performance on 1-back vs. 2-back tasks (Supplementary Table S1; Fig. 3a, b).

The left VLPFC, left DLPFC, and paracingulate/dACC brain regions showed greater activation during 1- vs. 2-back task anticipation across all emotional conditions (Supplementary Table S1; Fig. 3a, b). These regions fully overlapped with the regions showing greater activation for 2- vs. 1-back during task performance. No cortical or subcortical region showed greater activation for 2-back vs. 1-back task during anticipation. The paracingulate/dACC region will be further referred as paracingulate as per Harvard-Oxford cortical structural atlas.

\section{Elastic-net regression}

Forty-six input variables (27 neuroimaging [ $\Delta$ cue, $\Delta$ task, and $\Delta$ cue $^{*} \Delta$ task interaction in the left VLPFC, left DLPFC, and paracingulate gyrus/dACC for fearful, happy, and neutral faces], nine behavioral $\left[\Delta R T, \Delta\right.$ accuracy, and $\Delta R T^{*} \Delta$ accuracy interaction for fearful, happy, and neutral faces], and ten clinical [measures of current depression, mania, and anhedonia, measures of lifetime depression, mania, mood instability, and irritability, and measures of state and trait anxiety and medication load]) were predictors in the multinomial elastic net.

Multinomial model. In the multinomial model, the diagnostic group membership (BD vs. MDD vs. HC) was best explained by 15 of 46 input variables (Table 2, Fig. 3c): all ten clinical variables, $\Delta c u e$ and $\Delta c_{c u}^{*} \Delta$ task interaction in the left DLPFC for fearful faces, $\Delta c u e$ and $\Delta c u e^{*} \Delta$ task interaction in the paracingulate for neutral faces, and $\Delta$ cue in the left VLPFC for neutral faces.

Binomial models. Elastic net with neuroimaging, behavioral, and clinical variables as input selected 9 of 46 variables that best explained the BD vs. MDD differences (Table 2): the same five neuroimaging variables as described above and four clinical variables (current depression severity, lifetime mania, instability, and irritability). A random forest with these nine variables as predictors classified BD vs. MDD with $85.4 \%$ accuracy $(p<0.001)$.

Elastic net with clinical-only variables as input selected four of ten variables: current depression severity, lifetime mania, instability, and irritability. A random forest with these 4 variables as predictors classified BD vs. MDD with $80.5 \%$ accuracy $(p=0.001)$.

Elastic net with neuroimaging-only variables as input selected 7 of 27 variables: $\Delta$ task in left DLPFC for fearful faces, $\Delta$ task in the left DLPFC for neutral faces, and the 5 neuroimaging variables selected by the multinomial model (Table 2 ). A random forest with these 4 variables as predictors classified BD vs. MDD with $70.7 \%$ accuracy $(p=0.007)$.

Post hoc comparisons of the neuroimaging variables selected by multinomial/binomial elastic-net models. A one-way ANOVA on the neuroimaging variables identified by the elastic-net models revealed a significant effect of group on $\Delta$ cue for fearful faces in the left DLPFC $(F(2,61)=5.7, p=0.005$, Fig. 3d). A significantly greater decrease in the left DLPFC activation during anticipation of fearful faces in 2-back vs. 1-back conditions was observed in depressed individuals with MDD vs. those with BD (difference = $0.1, p$-adjusted $=0.004$ in the Tukey multiple comparison test), and a marginally greater decrease in MDD vs. $\mathrm{HC}$ (difference $=$ $0.06, p$-adjusted $=0.09$ ). There was a marginally significant effect of group on $\Delta$ cue for neutral faces in the left $\operatorname{VLPFC}(F(2,61)=2.8$, $p=0.07)$ and paracingulate gyrus $(\mathrm{F}(2,61)=2.6, p=0.08$, Fig. $3 \mathrm{~d})$. Individuals with $\mathrm{BD}$ showed a marginally greater decrease in activation in these regions during anticipation of neutral faces in 2-back vs. 1-back tasks compared with individuals with MDD (VLPFC: difference $=0.08, p$-adjusted $=0.06$; paracingulate: difference $=0.077, p$-adjusted $=0.076$ ).

The group differences among the regression slopes for $\Delta c u{ }^{*} \Delta$ task interaction effect in the left DLPFC for fearful faces and paracingulate gyrus for neutral faces (Fig. 3e) were examined using the R package "Ismeans" (https://cran.r-project.org/web/ packages/Ismeans/lsmeans.pdf). A significant interaction was observed in $\operatorname{DLPFC}(F(2,58)=3.86, p=0.03)$ and paracingulate $(\mathrm{F}(2,58)=4.4, p=0.01)$. A post hoc test with $p$-values adjusted using Tukey method for comparing a family of 3 estimates showed significant difference in slopes for BD vs. MDD (DLPFC: BD $(B=0.4)>M D D(B=-0.24)$, t.ratio $=2.6$, and $p$-adjusted $=0.03$; paracingulate: $\mathrm{BD}(\mathrm{B}=-0.57)<\mathrm{MDD}(\mathrm{B}=0.32)$, t.ratio $=-2.97$, and $p$-adjusted $=0.01$ ). Specifically, greater decreases in left DLPFC activation during anticipation of fearful faces for 2-back vs. 1-back task (positive values on the $x$-axis of Fig. 3e) were related to greater increases in the same region during performance on 2- vs. 1-back tasks (negative values on the y axis of Fig. 3e) in individuals with MDD, but an opposite pattern characterized individuals with BD. Greater decreases in paracingulate activation during anticipation of neutral faces for 2- vs. 1-back task were related to greater increases in the same region during performance on 2-back vs. 1 -back tasks in individuals with BD, but an opposite activation pattern characterized individuals with MDD.

\section{DISCUSSION}

Individuals with mood disorders often suffer from cognitive dysfunction [12-19] and aberrant anticipatory processing [25, 26]. Here we examined for the first time how the differences in brain activation during anticipation of and performance on an easy vs. 
A.

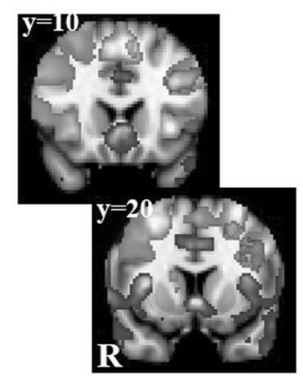

B.

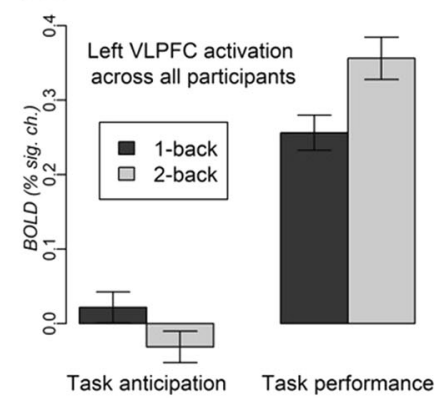

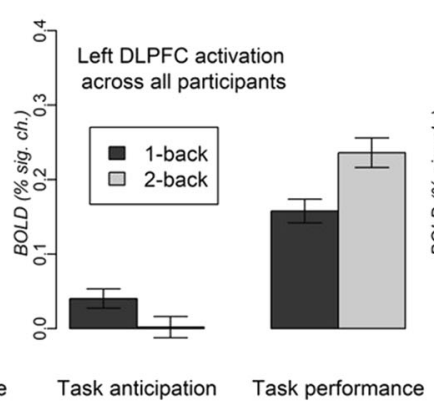

Task anticipation Task performance

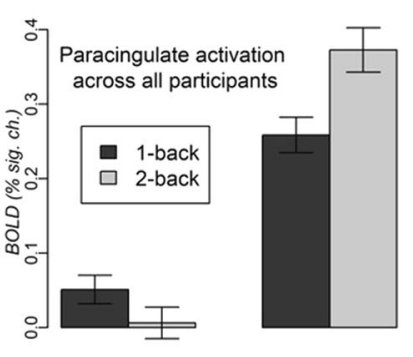

Task anticipation Task performance
C.

D.

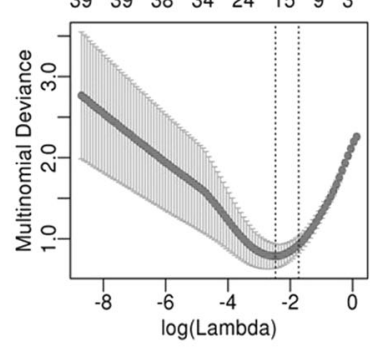

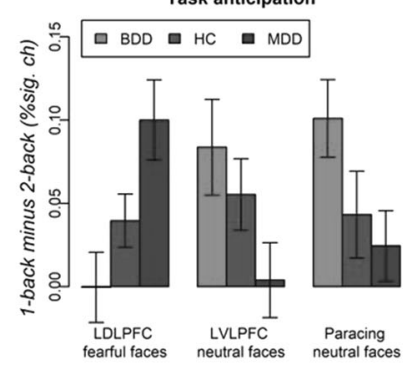

E.

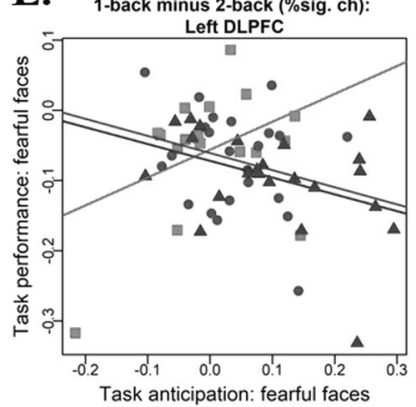

1-back minus 2-back (\%sig. ch):

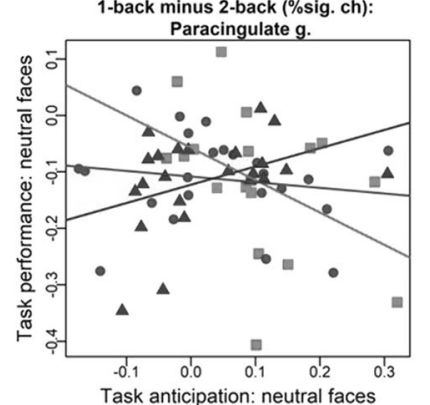

Fig. 3 Neuroimaging and elastic-net regression results. a Red-yellow: 2-back>1-back for task performance; blue: 1-back $>2$-back for task performance; green: 1-back>2-back for task anticipation. b Activation for 1- and 2-back task anticipation and task performance. c Crossvalidation plots for optimal lambda. $\mathbf{d} \Delta \mathbf{c u e}$ for the regions and conditions identified by the elastic-net regression analysis. e The interaction effect between $\Delta$ cue and $\Delta$ task in the left DLPFC for fearful faces, and in the paracingulate gyrus for neutral faces.

difficult emotional working memory tasks can help distinguish depressed individuals with BD from those with MDD. Given the nature of the task, we focused our analyses on the conjunction between the working memory network (i.e., the regions showing greater activation for difficult vs. easy tasks) and the network of regions involved in anticipation of easy vs. difficult tasks. The results pertaining to the regions identified in the conjunction analysis suggest that activation patterns in lateral and medial PFC during anticipation of easy vs. difficult tasks maybe an important biological marker for BD vs. MDD classification. Both multinomial (BD vs. $M D D$ vs. $H C$ ) and binomial (BD vs. MDD) elastic-net analyses showed that the 1-minus-2-back differences in the left DLPFC for fearful faces and the left VLPFC and paracingulate/dACC for neutral faces during task anticipation, as well as the interaction between these differences for task anticipation and task performance contributed to group classification. BD vs. MDD groups were classified with $85.4 \%$ accuracy based on clinical and neuroimaging variables selected by elastic net, with $80.5 \%$ accuracy based on clinical variables, and with $70.7 \%$ accuracy solely based on neuroimaging variables: the 1-minus-2-back differences in the left DLPFC, VLPFC, and paracingulate/dACC activation during anticipation of, performance on, and the interaction between anticipation and performance on working memory tasks with fearful and neutral faces. The fact that the direct comparisons between BD vs. HC or MDD vs. HC did not reach significance, but $\mathrm{BD}$ was significantly different from $M D D$, underscores the biologic differences between $B D$ and $M D D$, pointing toward the need for different and individualized treatment approaches.

Brain activation during task anticipation is related to expected difficulty and the amount of effort in the task [7, 41, 56], and emotional valence of the stimuli $[10,44,57]$. The left VLPFC is involved in cognitive control, memory retrieval, resolution of proactive interference $[58,59]$, and updating of the task-related representations prior to task performance [60]. Paracingulate/ $\mathrm{dACC}$ is a key region for conflict or interference monitoring [61], and may be involved with an effort calculation prior to task performance [56]. During task anticipation, these regions may work together to control neural and cognitive resource allocation to help achieve high-level performance on expected working memory. We found that individuals with BD who decreased paracingulate/dACC activation during anticipation of a difficult working memory task with neutral faces, increased it during task performance. In contrast, individuals with MDD who showed difficulty-related decreases in this region during task anticipation, failed to do so during task performance. Neutral faces are ambiguous for depressed individuals, and are often incorrectly judged as emotional $[62,63]$. Anticipation of neutral faces might engage different neural mechanisms in BD and MDD. While the former might employ a "resource saving" strategy for a more difficult task with neural faces, the latter might need to maintain or even increase anticipatory activation in paracingulate/dACC to be able to activate this region during the task.

The DLPFC plays a critical role in emotion regulation, cognitive control, and planning [64-66]. In our study, individuals with MDD, compared with those with $\mathrm{BD}$, had a significantly greater reduction in DLPFC activation for 2-back vs. 1-back anticipation of fearful faces, and had significantly more negative slope for the $\Delta c u e^{*} \Delta$ task interaction that did not differ from that in HC. The left DLPFC has been implicated in an early stage of threat processing [67], so failing to reduce activation in this region during anticipation of fearful faces in a difficult (2-back) working memory task might suggest aberrant anticipatory processing of negative stimuli in BD. This idea is consistent with our previous findings, suggesting that individuals with $B D$ may "block" anticipation of negative stimuli to avoid negative emotions prior to task performance [29, 68]. Anticipating a stressful day in the morning may negatively affect cognitive functioning during the day [69], so "blocking out" potentially stressful or disturbing information before experiencing it may be a defense mechanism that depressed individuals with BD use to remain functional. 
According to previous studies, the left VLPFC and dACC increase in activation for a more difficult, compared with an easy working memory task, but decrease in activation during anticipation of a more difficult, compared with an easy working memory task $[7,8]$. It is noteworthy that despite using a different subject population, different type of stimuli, different fMRI acquisition sequences, and analysis pipelines, we have replicated both the brain activation patterns for easy vs. difficult working memory task anticipation and performance, and the specific brain regions showing this pattern of activation [7]. This replication is critically important because it confirms the validity, reliability, and generalizability for the role of these regions during working memory task anticipation.

One limitation of our study is a relatively small sample size that did not allow us to examine separately or compare individuals with BD-I and BD-II. It is possible that the results were driven primarily by the BD-II as they comprised $80 \%$ of our BD sample. Our supplementary results, where we directly compared BD-II and MDD, provide additional support for this hypothesis (Supplementary Table S2). Another limitation is use of medications by some individuals with BD and MDD. However, it is important to note that a medication load was not different between BD and MDD groups, and did not contribute to BD vs. MDD classification.

In summary, our findings contribute to the body of literature, highlighting the role of lateral and medial PFC regions in distinguishing $B D$ from $\operatorname{MDD}[2,21,24,27]$. We showed that the 1-back vs. 2-back activation differences in these regions during anticipation of fearful and neutral faces distinguished BD from MDD when participants were depressed and thus especially difficult to diagnose. This brings anticipatory processing into focus, and suggests that anticipatory brain activation preceding performance on working memory tasks may be an important biological marker of BD and MDD. Differential aberrant anticipatory processing in individuals with BD and MDD might reflect the differences in mental strategies used to prepare to process fearful and neutral faces when neurocognitive resources are limited (as in a difficult working memory task) through changing the neural resource allocation prior to task performance. Targeting anticipatory processing may be a promising direction in developing therapies for BD and MDD. Future research should aim to understand how modulating PFC activation during mental preparation for difficult working memory tasks improves cognitive functioning in depressed individuals with BD and those with MDD.

\section{FUNDING AND DISCLOSURE}

This work was supported by a grant from the National Institute of Health K01MH104348 to A.M. A.M. and S.I. declare no conflict of interest. H.A.S. receives royalties from Wolters Kluwer, a grant from Myriad Genetics, and an editorial stipend from APA Press. M.L.P. received one honorarium from Sunovion Pharmaceuticals in the last 3 years.

\section{ACKNOWLEDGEMENTS}

The authors thank the participants for taking part in this research study

\section{ADDITIONAL INFORMATION}

Supplementary Information accompanies this paper at (https://doi.org/10.1038/ s41386-020-0638-7).

Publisher's note Springer Nature remains neutral with regard to jurisdictional claims in published maps and institutional affiliations.

\section{REFERENCES}

1. Hirschfeld RMA, Lewis L, Vornik LA. Perceptions and impact of bipolar disorder: how far have we really come? Results of the national depressive and manic-depressive association 2000 survey of individuals with bipolar disorder. J Clin Psychiatry. 2003:64:161-74.

2. de Almeida JR, Phillips ML. Distinguishing between unipolar depression and bipolar depression: current and future clinical and neuroimaging perspectives. Biol Psychiatry. 2013;73:111-8.

3. Baddeley A. Working memory. Curr Biol. 2010;20:R136-40.

4. Owen AM, McMillan KM, Laird AR, Bullmore E. N-back working memory paradigm: a meta-analysis of normative functional neuroimaging studies. Hum Brain Mapp. 2005;25:46-59.

5. Jonides J, Schumacher EH, Smith EE, Lauber EJ, Awh E, Minoshima S, et al. Verbal working memory load affects regional brain activation as measured by PET. J Cogn Neurosci. 1997;9:462-75.

6. Nystrom LE, Braver TS, Sabb FW, Delgado MR, Noll DC, Cohen JD. Working memory for letters, shapes, and locations: $\mathrm{AMRI}$ evidence against stimulus-based regional organization in human prefrontal cortex. Neuroimage. 2000;11:424-46.

7. Manelis A, Reder LM. He who is well prepared has half won the battle: an FMRI study of task preparation. Cereb Cortex. 2015;25:726-35.

8. Manelis A, Reder LM. Effective connectivity among the working memory regions during preparation for and during performance of the n-back task. Front Hum Neurosci. 2014. https://doi.org/10.3389/fnhum.2014.00593.

9. Curtis $C E$, D'Esposito $M$. The effects of prefrontal lesions on working memory performance and theory. Cogn Affect Behav Neurosci. 2004;4:528-39.

10. Ueda K, Okamoto Y, Okada G, Yamashita H, Hori T, Yamawaki S. Brain activity during expectancy of emotional stimuli: an fMRI study. Neuroreport. 2003;14:51-5.

11. Sohn M-H, Albert MV, Jung K, Carter CS, Anderson JR. Anticipation of conflict monitoring in the anterior cingulate cortex and the prefrontal cortex. Proc Natl Acad Sci USA. 2007;104:10330-4.

12. Christopher G, MacDonald J. The impact of clinical depression on working memory. Cogn Neuropsychiatry. 2005;10:379-99.

13. Clark L, Iversen SD, Goodwin GM. Sustained attention deficit in bipolar disorder. Br J Psychiatry. 2002;180:313-9.

14. Gohier B, Ferracci L, Surguladze SA, Lawrence E, El Hage W, Kefi MZ, et al. Cognitive inhibition and working memory in unipolar depression. J Affect Disord. 2009;116:100-5.

15. Harvey P-O, Fossati P, Pochon J-B, Levy R, Lebastard G, Lehéricy S, et al. Cognitive control and brain resources in major depression: an $\mathrm{fMRI}$ study using the $n$-back task. Neuroimage. 2005;26:860-9.

16. Lee $C-Y$, Wang L-J, Lee $Y$, Hung C-F, Huang $Y-C$, Lee $M-I$, et al. Differentiating bipolar disorders from unipolar depression by applying the brief assessment of cognition in affective disorders. Psychol Med. 2018;48:929-38.

17. Marazziti D, Consoli G, Picchetti M, Carlini M, Faravelli L. Cognitive impairment in major depression. Eur J Pharm. 2010;626:83-6.

18. Roca M, Vives M, López-Navarro E, García-Campayo J, Gili M. Cognitive impairments and depression: a critical review. Actas Esp Psiquiatr. 2015;43:187-93.

19. Taylor Tavares JV, Clark L, Cannon DM, Erickson K, Drevets WC, Sahakian BJ. Distinct profiles of neurocognitive function in unmedicated unipolar depression and bipolar II depression. Biol Psychiatry. 2007;62:917-24.

20. Almeida JRC, Mourao-Miranda J, Aizenstein HJ, Versace A, Kozel FA, Lu H, et al. Pattern recognition analysis of anterior cingulated cortex blood flow to classify depression polarity. Br J Psychiatry. 2013;203:310-1.

21. Delvecchio G, Fossati $P$, Boyer $P$, Brambilla $P$, Falkai $P$, Gruber $O$, et al. Common and distinct neural correlates of emotional processing in Bipolar Disorder and Major Depressive Disorder: a voxel-based meta-analysis of functional magnetic resonance imaging studies. Eur Neuropsychopharmacol. 2012;22:100-13.

22. Fournier JC, Keener MT, Almeida J, Kronhaus DM, Phillips ML. Amygdala and whole-brain activity to emotional faces distinguishes major depressive disorder and bipolar disorder. Bipolar Disord. 2013;15:741-52.

23. Townsend J, Altshuler LL. Emotion processing and regulation in bipolar disorder: a review. Bipolar Disord. 2012;14:326-39.

24. Zhu Y, Quan W, Wang H, Ma Y, Yan J, Zhang $\mathrm{H}$, et al. Prefrontal activation during a working memory task differs between patients with unipolar and bipolar depression: a preliminary exploratory study. J Affect Disord. 2018;225:64-70.

25. Andersen SM, Spielman LA, Bargh JA. Future-event schemas and certainty about the future: automaticity in depressives' future-event predictions. J Pers Soc Psychol. 1992;63:711-23.

26. Strunk DR, Lopez H, DeRubeis RJ. Depressive symptoms are associated with unrealistic negative predictions of future life events. Behav Res Ther. 2006;44: 861-82.

27. Bertocci MA, Bebko GM, Mullin BC, Langenecker SA, Ladouceur CD, Almeida JRC, et al. Abnormal anterior cingulate cortical activity during emotional n-back task performance distinguishes bipolar from unipolar depressed females. Psychol Med. 2012;42:1417-28.

28. Chase HW, Nusslock R, Almeida JR, Forbes EE, LaBarbara EJ, Phillips ML. Dissociable patterns of abnormal frontal cortical activation during anticipation of 
an uncertain reward or loss in bipolar versus major depression. Bipolar Disord. 2013;15:839-54.

29. Manelis A, Almeida JRC, Stiffler R, Lockovich JC, Aslam HA, Phillips ML. Anticipation-related brain connectivity in bipolar and unipolar depression: a graph theory approach. Brain. 2016;139:2554-66.

30. Roiser JP, Sahakian BJ. Hot and cold cognition in depression. CNS Spectr. 2013;18:139-49.

31. Zou $\mathrm{H}$, Hastie T. Regularization and variable selection via the Elastic Net. J Royal Stat Soc. 2005;67:301-20.

32. Evans JS, Murphy MA, Holden ZA, Cushman SA. Modeling Species Distribution and Change Using Random Forest. In: Drew C, Wiersma Y, Huettmann F (eds). Predictive Species and Habitat Modeling in Landscape Ecology. New York, NY: Springer; 2011.

33. Hamilton M. A rating scale for depression. J Neurol Neurosurg Psychiatry 1960;23:56-62.

34. Sheehan DV. MINI-Mini International neuropsychiatric interview-english version 5.0. 0-DSM-IV. J Clin Psychiatry. 1998;59:34-57.

35. Blair JR, Spreen O. Predicting premorbid IQ: a revision of the national adult reading test. Clin Neuropsychol. 1989;3:129-36.

36. Young RC, Biggs JT, Ziegler VE, Meyer DA. A rating scale for mania: reliability, validity and sensitivity. Br J Psychiatry. 1978;133:429-35.

37. Dell'Osso L, Armani A, Rucci P, Frank E, Fagiolini A, Corretti G, et al. Measuring mood spectrum: comparison of interview (SCI-MOODS) and self-report (MOODSSR) instruments. Compr Psychiatry. 2002;43:69-73.

38. Spielberger CD, Gorsuch RL, Lushene R, Vagg PR, Jacobs GA. Manual for the StateTrait Anxiety Inventory. Palo Alto, CA: Consulting Psychologists Press; 1983.

39. Snaith RP, Hamilton M, Morley S, Humayan A, Hargreaves D, Trigwell P. A scale for the assessment of hedonic tone the Snaith-Hamilton Pleasure Scale. Br J Psychiatry. 1995;167:99-103.

40. Hassel S, Almeida JR, Kerr N, Nau S, Ladouceur CD, Fissell K, et al. Elevated striatal and decreased dorsolateral prefrontal cortical activity in response to emotional stimuli in euthymic bipolar disorder: no associations with psychotropic medication load. Bipolar Disord. 2008;10:916-27.

41. Manelis A, Reder LM. Effective connectivity among the working memory regions during preparation for and during performance of the n-back task. Front Hum Neurosci. 2014;8:593.

42. Tottenham N, Tanaka JW, Leon AC, McCarry T, Nurse M, Hare TA, et al. The NimStim set of facial expressions: judgments from untrained research participants. Psychiatry Res. 2009;168:242-9.

43. Goeleven E, De Raedt R, Leyman L, Verschuere B. The Karolinska directed emotional faces: a validation study. Cogn Emot. 2008;22:1094-118.

44. Abler B, Erk $\mathrm{S}$, Herwig $\mathrm{U}$, Walter $\mathrm{H}$. Anticipation of aversive stimuli activates extended amygdala in unipolar depression. J Psychiatr Res. 2007;41:511-22.

45. Esteban O, Birman D, Schaer M, Koyejo OO, Poldrack RA, Gorgolewski KJ. MRIQC: advancing the automatic prediction of image quality in MRI from unseen sites. PLoS ONE. 2017;12:e0184661.

46. Esteban O, Markiewicz CJ, Blair RW, Moodie CA, Isik Al, Erramuzpe A, et al. fMRIPrep: a robust preprocessing pipeline for functional MRI. Nat Methods. 2019;16:111-6.

47. Dale AM, Fischl B, Sereno MI. Cortical surface-based analysis. I. Segmentation and surface reconstruction. Neuroimage. 1999;9:179-94.

48. Zhang Y, Brady M, Smith S. Segmentation of brain MR images through a hidden Markov random field model and the expectation-maximization algorithm. IEEE Trans Med Imaging. 2001;20:45-57.
49. Cox RW, Hyde JS. Software tools for analysis and visualization of fMRI data. NMR Biomed. 1997;10:171-8.

50. Greve DN, Fischl B. Accurate and robust brain image alignment using boundarybased registration. Neuroimage. 2009;48:63-72.

51. Jenkinson M, Bannister P, Brady M, Smith S. Improved optimization for the robust and accurate linear registration and motion correction of brain images. Neuroimage. 2002;17:825-41.

52. Pruim RHR, Mennes $M$, van Rooij D, Llera A, Buitelaar JK, Beckmann CF. ICAAROMA: A robust ICA-based strategy for removing motion artifacts from fMRI data. Neuroimage. 2015;112:267-77.

53. Winkler AM, Ridgway GR, Webster MA, Smith SM, Nichols TE. Permutation inference for the general linear model. Neuroimage. 2014;92:381-97.

54. Lockhart R, Taylor J, Tibshirani RJ, Tibshirani R. A significance test for the LASSO. Ann Stat. 2014;42:413-68.

55. Taylor J, Tibshirani RJ. Statistical learning and selective inference. Proc Natl Acad Sci USA. 2015;112:7629-34.

56. Croxson PL, Walton ME, O'Reilly JX, Behrens TEJ, Rushworth MFS. Effort-based cost-benefit valuation and the human brain. J Neurosci. 2009;29:4531-41.

57. Herwig U, Baumgartner T, Kaffenberger T, Brühl A, Kottlow M, Schreiter-Gasser U, et al. Modulation of anticipatory emotion and perception processing by cognitive control. Neuroimage. 2007;37:652-62.

58. Badre $D$, Wagner AD. Left ventrolateral prefrontal cortex and the cognitive control of memory. Neuropsychologia. 2007;45:2883-901.

59. Oztekin I, Badre D. Distributed patterns of brain activity that lead to forgetting. Front Hum Neurosci. 2011;5:86.

60. Brass $M$, von Cramon DY. Selection for cognitive control: a functional magnetic resonance imaging study on the selection of task-relevant information. J Neurosci. 2004;24:8847-52.

61. Bush G, Shin LM, Holmes J, Rosen BR, Vogt BA. The Multi-Source Interference Task: validation study with $\mathrm{fMRI}$ in individual subjects. Mol Psychiatry. 2003;8:60.

62. Leppänen JM, Milders M, Bell JS, Terriere E, Hietanen JK. Depression biases the recognition of emotionally neutral faces. Psychiatry Res. 2004;128:123-33.

63. Manelis A, Huppert T, Rodgers E, Swartz HA, Phillips ML. The role of the right prefrontal cortex in recognition of facial emotional expressions in depressed individuals: fNIRS study. J Affect Disord. 2019;258:151-8.

64. Barbey AK, Koenigs M, Grafman J. Dorsolateral prefrontal contributions to human working memory. Cortex. 2013;49:1195-205.

65. Kaller CP, Rahm B, Spreer J, Weiller C, Unterrainer JM. Dissociable contributions of left and right dorsolateral prefrontal cortex in planning. Cereb Cortex 2011;21:307-17.

66. Fellows LK, Farah MJ. Different underlying impairments in decision-making following ventromedial and dorsolateral frontal lobe damage in humans. Cereb Cortex. 2005;15:58-63.

67. Sagliano L, D'Olimpio F, Panico F, Gagliardi S, Trojano L. The role of the dorsolateral prefrontal cortex in early threat processing: a TMS study. Soc Cogn Affect Neurosci. 2016;11:1992-8.

68. Manelis A, Stiffler R, Lockovich JC, Almeida JRC, Aslam HA, Phillips ML. Longitudinal changes in brain activation during anticipation of monetary loss in bipolar disorder. Psychol Med. 2019;49:2781-8.

69. Hyun J, Sliwinski MJ, Smyth JM. Waking up on the wrong side of the bed: the effects of stress anticipation on working memory in daily life. J Gerontol B Psychol Sci Soc Sci. 2019;74:38-46. 